Revista Aportes para la Integración Latinoamericana Año XXIV, N 38/Junio 2018, ISSN 2468-9912. DNDA: 5355295 en línea Milena Olivares Homez

El consejo de Salud Suramericano y la creación de un régimen regional de salud

Págs. 16-44

\title{
El Consejo de Salud SuRamericano y la creación de un RÉgimen REGIONAL DE SALUD
}

\section{The South American Health Council and the creation of a Regional HEALTH REGIME}

Milena Olivares Homez

\begin{abstract}
RESUMEN
Este artículo aborda los principales postulados de los regímenes internacionales desde la teoría neoliberal y los aplica al caso de estudio del Consejo de Salud Sudamericano (CSS), de la UNASUR, analizando si los desarrollos de este último pueden considerarse como un régimen regional de salud. Se revisa detalladamente el proceso de institucionalización del consejo y de sus organismos subsidiarios con una metodología cualitativa, cuyas fuentes primarias son textos, acuerdos fundacionales y las entrevistas a personas relacionadas con el tema. Es importante el trabajo del CSS recopilando información del estado de la salud regional y contribuyendo en la toma de decisiones de los ministros de salud para mejorar el acceso a la salud de los suramericanos.

Esta investigación argumenta que la dinámica que ha creado el CSS especialmente con la instauración del Instituto Suramericano de Gobierno en Salud, ha generado ciertas características de los regímenes internacionales. Sin embargo, en medio de esta sinergia no aparece una función fundamental de los regímenes y que es esencial para su mantenimiento, a saber: cualquier forma de obligación hacia los estados para que cumplan los acuerdos y de esta manera todos los miembros aumenten la confianza hacia el régimen.
\end{abstract}

\section{PALABRAS CLAVE}

UNASUR, Consejo de Salud Suramericano, ISAGS, Régimen Internacional, Regionalismo Post Hegemónico

\section{ABSTRACT}

This article addresses the main postulates of international regimes from the neoliberal theory and applies them to the case study of the South American Health Council (CSS By its initials in Spanish), of UNASUR, analyzing whether the developments of the latter can be considered as a regional health regime. The institutionalization process of the council and its subsidiary organisms are reviewed in detail with a qualitative methodology, whose primary sources are texts, foundational agreements and the interviews with people related to the topic. It is important the work of the CSS collecting information about the regional health and contributing to the decision making of health ministers in order to improve access to health for South Americans. This research argues that the dynamic that the CSS has created, especially with the establishment of the South American Institute of Government in Health, has generated certain characteristics of the international regimes. However, in the middle of this synergy does not appear a fundamental function of the regimes, and that is essential for its maintenance, namely: any way in which they can make it mandatory for the States to follow the agreements and in this way all the members increase the confidence towards the regime.

\section{KEY WORDS}

Union of South American Nations (UNASUR), South American Health Council, South American Institute of Government in Health (ISAGS), International Regime, Post-Hegemonic Regionalism 
Revista Aportes para la Integración Latinoamericana Año XXIV, Nº 38/Junio 2018, ISSN 2468-9912. DNDA: 5355295 en línea

Milena Olivares Homez

El consejo de Salud Suramericano y la creación de un régimen regional de salud

Págs. 16-44

\section{INTRODUCCIÓN}

La Unión de Naciones Suramericanas (UNASUR) nació como un esfuerzo conjunto de los países de América del Sur para crear un espacio de discusión política e integración regional. Los primeros esfuerzos se remontan a 2004 cuando se creó la Comunidad Suramericana de Naciones (CSN) y las posteriores reuniones que se hicieron entre los países hasta 2007. En ese año en que se decide cambiar el nombre de la CSN por el de UNASUR en mayo del 2008 se aprueba el Tratado Constitutivo, que entra en vigencia el 11 de marzo de 2011.

El tema de la UNASUR ha sido objeto de estudio de la integración ${ }^{1}$ y el regionalismo ${ }^{2}$. Así, unos lo califican como uno más de los intentos fallidos de la región por lograr una integración al estilo de la Unión Europea (Malamud y Gardini, 2012), otros por el contrario lo asumen como un proceso insigne de la nueva ola regionalista que atraviesa la región y que se desmarca de los anteriores procesos, por el protagonismo de los temas sociales y el retorno del Estado en la conducción de los procesos de integración. Además el tema económico deja de ser el eje de la integración y la cooperación se amplía a sectores como salud, educación, medio ambiente, entre otros (Sanahuja, 2012; Riggirozzi y Tussie, 2012, Briceño Ruiz, 2014).

La UNASUR tiene una Secretaria General y tres órganos con diferentes niveles de decisión, así su máxima instancia es el Consejo de Jefas y Jefes de Estados y de Gobierno, seguido por el Consejo de Ministras y Ministros de Relaciones Exteriores y

\footnotetext{
${ }^{1}$ En la región la integración tradicionalmente ha sido analizada básicamente en términos económicos, incluyendo los enfoques que toman como modelo la Unión Europea o aquellos que hablan de regionalismo enmarcado en la idea de fortalecer las instituciones y acuerdos regionales que mejoren la productividad comercial entre países contiguos. Haas (1971, p.6), propone la integración regional como un proceso en que los estados nacionales "se mezclan, confunden y fusionan voluntariamente con sus vecinos de modo tal que pierden ciertos atributos fácticos de soberanía, a la vez que adquieren nuevas técnicas para resolver conjuntamente sus conflictos". Las etapas tradicionales de la integración son: zona de libre comercio, mercado común, unión aduanera, unión económica y la comunidad económica" Balassa (1964, p.2), aquí cada nivel superior integra las desregulaciones ejecutadas en el nivel anterior.

${ }^{2}$ Otros autores, en cambio, prefieren analizar los fenómenos como UNASUR desde la óptica del regionalismo, señalando que "el concepto de regionalismo es más amplio que el de integración económica regional y se entiende como un proceso consciente de diseño y aplicación de políticas comunes o coordinadas para reorganizar un espacio geográfico regional determinado" (Sanahuja 2008-2009, p.7). Este mismo autor sostiene que la región ha pasado de un regionalismo abierto a un regionalismo post-liberal, señala que hay un "agotamiento del ciclo de 'regionalismo abierto', cuyo eje fundamental es el intercambio económico y que para América Latina representaron los procesos de la Comunidad Andina de Naciones (CAN) y el Mercado Común del Sur (MERCOSUR)" (Sanahuja 2008-2009, p.11). Así, el autor inscribe las propuestas de la UNASUR, el ALBA y la CELAC, como expresión del regionalismo post liberal.
} 
Revista Aportes para la Integración Latinoamericana Año XXIV, N³8/Junio 2018, ISSN 2468-9912. DNDA: 5355295 en línea

Milena Olivares Homez

El consejo de Salud Suramericano y la creación de un régimen regional de salud

Págs. 16-44

finalmente el Consejo de Delegados y Delegadas. La UNASUR está compuesta por doce consejos ministeriales y sectoriales, dentro de estos consejos se encuentra el Consejo de Salud Suramericano (CSS), que nació en 2008 por decisión de las Jefas y Jefes de Estado. El CSS decide conformar en 2009 los Grupos Técnicos (GT) y las Redes Estructurantes y en 2011 el Instituto Suramericano de Gobierno en Salud; estos tres órganos subsidiarios fueron fundamentales para que el Consejo comenzara a trabajar el tema de la salud en la región y avanzara en algunas líneas de investigación sobre salud Pública.

El proceso de UNASUR, específicamente en el tema de la salud ha sido estudiado desde la gobernanza regional (Riggirozzi, 2012a), con el ánimo de mostrar los avances que ha tenido este proceso y que no se pueden simplemente dejar de lado por no estar dentro de alguna de las etapas tradicionales de la integración. UNASUR-Salud ha sido una interesante propuesta que convocó a los gobiernos de toda la región y, que a pesar de los cambios ideológicos de algunos de ellos los proyectos de salud regional siguen funcionando. Este artículo analiza las acciones y logros de UNASUR en materia de salud a través del estudio de la posible formación de un régimen regional ${ }^{3}$, asunto que se constituiría como el mayor de los logros de esta organización. El tema del régimen regional no ha estado presente en los análisis de los estudiosos, por lo tanto, ver la salud desde el marco regional, permite revisar en detalle todas las propuestas y proyectos que se dieron al interior de la UNASUR y, el papel que ha jugado la institucionalidad a la hora de concretar acuerdos y alcanzar metas comunes.

Lo que se ha dado en la región en materia de salud a partir de la materialización de los objetivos del CSS, la creación de las Redes Estructurantes (RES), los Grupos Técnicos (GT) y el Instituto Suramericano de Gobierno en Salud (ISAGS), permite pensar en la configuración de un régimen regional. La mayoría de todos los procesos y actuaciones

\footnotetext{
${ }^{3}$ Un régimen internacional es un conjunto de normas, reglas y procedimientos de toma de decisión (Krasner 1982a, p.186) alrededor del cual convergen las expectativas de varios actores, en un tema específico. Por su parte, la región es definida de varias maneras y dependiendo del área del conocimiento. Para el caso que nos atañe, desde el punto de vista de las Relaciones Internacionales las regiones son vistas como "subsistemas del sistema internacional o formaciones regionales emergentes con sus propias dinámicas" (Hettne 2006, p.2). Los países de este subsistema comparten proyectos políticos y económicos y tienen un cierto grado de identidad común. En este entendido un régimen regional es ese conjunto de normas, reglas y procedimientos que es compartido por países que tienen una proximidad geográfica y en cierta medida identitaria, como es el caso de América del Sur.
} 

Año XXIV, N 38/Junio 2018, ISSN 2468-9912. DNDA: 5355295 en línea

Milena Olivares Homez

El consejo de Salud Suramericano y la creación de un régimen regional de salud

Págs. 16-44

que se han dado en el CSS se entienden a la luz de los regímenes internacionales. Sin embargo, hasta ahora no se observa una característica fundamental de los mismos y que tiene que ver con la capacidad de persuasión que obligue a los estados a cumplir lo pactado. La evaluación final sobre la existencia o no de un régimen regional en salud dependerá no solo de esta última característica, porque en efecto el tema de la salud en la UNASUR ha generado ciertas sinergias y características que bien caben en la teoría de los regímenes internacionales. La metodología utilizada en esta investigación es de carácter cualitativo y el método es el caso de estudio porque permite evidenciar la configuración específica de los factores que llevaron a un resultado observado y esta investigación pretende evidenciar si se observa en la región un régimen regional de salud o no. Como unidades de análisis se proponen el CSS, el ISAGS, Grupos Técnicos y Redes Estructurantes. Teniendo en cuenta que las principales estrategias para la recolección de información cualitativa son las entrevistas, esta investigación utiliza cuatro entrevistas semiestructuradas que fueron practicadas a actores del proceso de salud en UNASUR y a académicos expertos en el tema. También se usaron estrategias de investigación y análisis documental con base en los documentos disponibles en internet, específicamente los textos fundacionales de la de la UNASUR, el CSS y el ISAGS. EI período de la investigación está comprendido entre 2007 y 2017.

El artículo está divido en tres partes, en la primera se aborda el marco teórico y se explica la teoría de los regímenes internacionales desde la visión neoliberal, que es la que más se ajusta al caso de estudio y adicionalmente da cuenta de dos conceptos que son fundamentales para entender esta teoría y el caso en cuestión: institucionalización y organizaciones internacionales. En la segunda parte se aborda la creación del CSS así como de sus organismos subsidiarios del CSS: RES, GT e ISAGS y se exponen los elementos que hacen parte del proceso de institucionalización de los mismos. En la tercera sección se discute si los desarrollos de UNASUR en materia de salud hacen parte de un régimen regional de salud, en comparación con la teoría neoliberal. Finalmente las conclusiones recogen los principales hallazgos de la investigación evidenciando que lo que se ha desarrollado en la UNASUR cumple con varias características de los regímenes internacionales, pese a su corta existencia.

\section{RegímENES INTERNACIONALES}


Revista Aportes para la Integración Latinoamericana Año XXIV, N 38/Junio 2018, ISSN 2468-9912. DNDA: 5355295 en línea

Milena Olivares Homez

El consejo de Salud Suramericano y la creación de un régimen regional de salud

Págs. 16-44

Los regímenes Internacionales han emergido como una forma de ayudar a los estados a manejar los temas más difíciles de la política mundial, que dicho sea de paso está cada vez más interconectada. "La política mundial se caracteriza por la interdependencia en seguridad: ni siquiera el Estado más poderoso, puede manejar esos problemas solo, el mundo de hoy requiere que estados y actores no estatales coordinen acciones, a menudo a través de organizaciones internacionales, para atender estos asuntos" (Frederking y Diehl, 2015, p.1).

Varios autores como Frederking y Diehl (2015), Robert Keohane y Nye (2012), Krasner (1982a), Young (1982) sostienen que a partir de la Segunda Guerra Mundial el mundo asiste a una reconfiguración política, que entiende los sucesos más allá de los enfoques que hablan de la distribución del poder. Se necesita entonces nuevos enfoques que reconozcan los nuevos actores, desafíos y amenazas en el plano internacional y así entender cómo se relacionan hoy los países y cómo arreglan sus diferencias para no recurrir a la violencia como última instancia. Es así como los actores del sistema internacional han desarrollado una serie de normas, reglas y procedimientos para trabajar en conjunto por sus objetivos en diversas áreas, este es el inicio de los regímenes internacionales.

En su acepción más aceptada los regímenes son definidos comúnmente como una variedad de acuerdos y normas que sirven para guiar el comportamiento de los estados que hacen parte del régimen y que dichos comportamientos responden a sus intereses compartidos; estos comportamientos deben "producir resultados colectivos que están en armonía con los objetivos y comparten las convicciones que están especificadas en los principios del régimen" (Hasenclever et al., 1997, p.9). Jervis (1982), Puchala y Hopkins (1982), Keohane (1982) sostienen que los regímenes influyen en el comportamiento de los estados. Estos acuerdos pueden institucionalizarse a través de la creación de organizaciones o instituciones, que les dan un aspecto más formal. La definición más conocida es la de Stephen Krasner, que los define como:

Conjunto implícito o explícito de normas, reglas y procedimientos de toma de decisiones alrededor de los cuales convergen las expectativas de los actores en un área dada de las relaciones internacionales. Los principios son creencias de hecho, causación y rectitud. Las normas son estándares de comportamiento definido en términos de derechos y obligaciones. Las reglas son prescripciones o proscripciones para la acción. Los procedimientos de toma de decisiones son prácticas que prevalecen para crear 0 implementar decisiones colectivas (Krasner, 1982a, p.186). 

Año XXIV, N 38/Junio 2018, ISSN 2468-9912. DNDA: 5355295 en línea

Milena Olivares Homez

El consejo de Salud Suramericano y la creación de un régimen regional de salud

Págs. 16-44

En la misma dirección Keohane y Nye proponen los regímenes como "un conjunto de arreglos gubemamentales que incluyen redes de reglas, normas y procedimientos que regularizan el comportamiento y controlan sus efectos" (Keohane y Nye, 2012, p.9). Los estudios sobre los regímenes pueden clasificarse en tres grandes escuelas: "la escuela realista basa sus análisis en las relaciones de poder, la escuela neoliberal en las constelaciones de interés y los cognitivistas enfatizan en las dinámicas de conocimiento, comunicación e identidades" (Hasenclever et al., 1997, pp.1-2). A continuación se explica más detalladamente la teoría de la segunda escuela.

\section{1. Teoría Neoliberal de los Regímenes Internacionales}

La premisa principal de la teoría neoliberal de los regímenes es que son creados por los intereses de los estados y justamente los ayudan a llevar a cabo sus intereses comunes. Esto no significa que pasen de lado los efectos de las diferencias de poder, pero ciertamente no las ponen en el centro del debate.

Los neoliberales ven a los estados como egoístas racionales que se preocupan por sus ganancias absolutas, de tal manera que han elaborado teorías económicas de las instituciones enfocándose en los costos de información y transacción. También adoptan una perspectiva institucionalista del sistema internacional y le asignan a los regímenes la función de ayudar a coordinar el comportamiento de los estados de tal modo que eviten resultados subóptimos a nivel colectivo y así se cumple con los intereses personales de los estados y se logra que estos crean que es necesario mantenerlos.

El aumento o profundización de la necesidad de mantener los regímenes es evidencia de que el mismo habrá cobrado vida propia y que incluso iría más allá de los factores (variables causales básicas, Krasner 1982b) que lo hicieron surgir. Los neoliberales también proponen "analizar los regímenes como un fenómeno estrictamente basado en el interés, la creación, mantenimiento y desaparición de tales pueden y deben ser tomados en cuenta desde la perspectiva de actores estratégicamente racionales pero por lo demás indiferentes entre sí "(Hasenclever et al., 1997, p.26).

El neoliberalismo institucional ve a los regímenes como arreglos o acuerdos particulares en áreas sustantivas de las relaciones internacionales. En muchos casos reflejan políticas de contingencia y a partir de estas se establecen regímenes de intereses comunes o de 

Año XXIV, N 38/Junio 2018, ISSN 2468-9912. DNDA: 5355295 en línea Milena Olivares Homez El consejo de Salud Suramericano y la creación de un régimen regional de salud

aversiones comunes. En los regímenes de intereses comunes, los actores están de acuerdo en que si cada uno juega su propia estrategia -así sea racional-, el resultado puede ser subóptimo para todos y, en este caso la estrategia de colaboración puede ser la política más óptima para adoptar.

En los regímenes de aversiones comunes "los actores no están de acuerdo en un resultado comúnmente preferido, pero están de acuerdo en el resultado que todos desean evitar; tales regímenes simplemente requieren coordinación de políticas, no colaboración. Se aceptan prohibiciones de conducta, no se requiere supervisión central y rara vez se persiguen políticas conjuntas" (Haas, 1982, pp. 211-212).

Los neoliberales piensan que los regímenes pueden ser muy eficaces si ocurre un cambio en las preferencias "los cambios en preferencias sobre las estrategias usualmente son suficientes para producir beneficios mutuos. Mucho de este cambio viene por más y mejor información, información acerca de la situación, información acerca de que ha hecho el otro lado y por qué lo ha hecho y, la información acerca de lo que probablemente el otro lado haga en el futuro" (Jervis, 1999, p.51). También se asume que los estados pueden cooperar si se reducen los costos de transacción, que son los costos y riesgos asociados con el alcance y cumplimiento de los acuerdos.

Con relación a los incentivos para crear los regímenes todas las teorías que abordan su estudio, comparten el análisis hecho por Keohane, quien sostiene que en el siglo XX se ha visto una expansión real y potencial de las confrontaciones en el plano internacional, de igual modo se han incrementado los intercambios y transacciones de todo tipo y en muchas áreas, (bienes, personas, mercado, tecnología, etc.), situaciones que generan un aumento de la interdependencia y por tanto de la necesidad de cooperación.

Para entender las funciones de los regímenes internacionales es necesario tener en cuenta dos características importantes del contexto internacional. La primera es que la política mundial carece de autoridades gubernamentales y la segunda es que justamente por la primera razón existe una incertidumbre penetrante. Así, dado el contexto internacional la función más importante de los regímenes internacionales es facilitar la creación de acuerdos mutuos entre gobiernos, de modo tal que la condición estructural de anarquía no lidere completamente (Keohane, 1982, p.332). 

Año XXIV, N 38/Junio 2018, ISSN 2468-9912. DNDA: 5355295 en línea Milena Olivares Homez El consejo de Salud Suramericano y la creación de un régimen regional de salud Págs. 16-44

La teoría neoliberal de los regímenes internacionales señala que, a raíz de la interdependencia, se incrementa la necesidad de realizar una coordinación política y esto conduce a una mayor cooperación. Del mismo modo sostiene que la cooperación bajo ciertas condiciones (que se desarrollan en la base de los intereses y de las instituciones), puede afectar los patrones de cooperación que emergen de las mismas y así incidir en los patrones de comportamiento de los actores que participan del régimen de cooperación (Keohane y Nye, 2012).

Para Keohane un incentivo bastante importante de los regímenes es que "las instituciones y procedimientos que se desarrollan alrededor de los regímenes internacionales, adquieren valor en cuanto a que son acuerdos que permiten la comunicación y por lo tanto facilitan el intercambio de la misma y esto es una prueba de la creciente demanda hacia ellos" (1982, p. 348). Los regímenes pueden adquirir vida propia y una vez establecidos pueden retroalimentar las variables causales básicas que le dieron origen (interés propio, poder político, conocimiento, entre otras); es más podrían alterar la distribución de poder y hacer cambios en las valoraciones de los intereses (Krasner, 1982b, p. 500).

Las ventajas informativas que proporcionan los principios, las normas, las reglas y los procedimientos de toma de decisiones existentes y que se derivan de la creación de los regímenes, permiten su supervivencia más allá de una posible fragmentación del poder Aggarwal (1981, p.16). En el mundo político los estados siempre están buscando reducir riesgos y conflictos de interés a través de la coordinación de su comportamiento, de modo tal que un acuerdo en un área pueda impactar en otras áreas dentro de un sistema multicapas o interconectado y se logre consolidar acuerdos en múltiples niveles y distintas áreas. En este contexto "la función más importante del régimen internacional es facilitar la creación de acuerdos específicos en materias muy importantes dentro de un área cubierta por el régimen" (Keohane, 1982, p.334).

Los regímenes tienen dos funciones muy importantes que evidentemente motivan su creación. La primera es establecer marcos de negociación a través de la reducción de los costos de transacción (que son los costos secundarios y los riesgos que se derivan de la creación y cumplimiento de los acuerdos), la segunda tiene que ver con la ayuda que 

Año XXIV, N 38/Junio 2018, ISSN 2468-9912. DNDA: 5355295 en línea

Milena Olivares Homez

El consejo de Salud Suramericano y la creación de un régimen regional de salud

Págs. 16-44

presta en la coordinación de expectativas de los actores por medio de la mejora en la calidad y la cantidad de información disponible para los estados.

Aunque el contexto político de la época era marcadamente antineoliberal en lo económico y en lo político, la teoría neoliberal de los regímenes internacionales se ajusta al estudio de caso porque, más allá de la retórica ideológica de los presidentes del momento, lo cierto es que todos tenían interés en mejorar el estado de la salud en sus países. En primer lugar, cada país tenía motivaciones diferentes, unos de forma más altruista pensaban incluso en la universalización del sistema de salud, mientras otros estaban más preocupados por el tema de la prevención, que podría ahorrar dinero al sistema de salud en el tratamiento de enfermedades.

En segundo lugar, desde la teoría neoliberal la formación de los regímenes puede darse por situaciones de contingencia y ese fue un elemento importante para la creación del CSS. En tercer lugar, los regímenes proveen marcos de negociación e información, situación que es ventajosa para los países de la región porque pueden compartir buenas prácticas en salud $\mathrm{y}$ hacer frente de manera mancomunada a los problemas transfronterizos en materia de salud. $\mathrm{Y}$, finalmente desde el neoliberalismo se sostiene que los países siempre están buscando reducir riesgos y el conflicto situación que concuerda con un principio rector de la UNASUR: la paz.

\section{1.1. Institucionalización y Organizaciones internacionales}

La teoría neoliberal sostiene que a partir de patrones institucionalizados o hábitos de cooperación en determinadas áreas temáticas se pueden generar regímenes internacionales, así Rochester (1975) los describe como un conjunto de acciones modeladas que colaboran para alcanzar formas de coordinación multilateral y cooperación. No obstante, los regímenes pueden estar o no, asistidos por acuerdos organizacionales (Young, 1982). El neoliberalismo institucional resalta el rol que juegan las instituciones, las cuales son consideradas como modelos de endurecimiento de perspectivas compartidas sobre la conducta y que han tenido cierto nivel de aprobación formal. Por lo anterior se señala que la instauración de instituciones puede conllevar al aumento de la cooperación (Jervis, 1999, pp. 53-54). 
Para esta investigación se comprenderá el término de institucionalización como la incorporación de las concepciones que los países tienen acerca de la salud en la región por medio de la creación de convenios para establecer -en primer lugar- el CSS, después las RES, los GT y el ISAGS. Asimismo, la ejecución de estos convenios se percibirá desde el establecimiento de lugares físicos y la contratación de una planta de personal, para que estos órganos lleven a cabo las tareas encomendadas y así cumplir los propósitos de Salud de la UNASUR. En consecuencia, todos los proyectos, estudios, planes y sugerencias que resulten de la labor de estos organismos serán concebidos como parte de la institucionalización que la UNASUR le ha otorgado al asunto de la Salud. Así, es evidente que la fundación de estos órganos o "arreglos organizacionales", es una cuestión trascendental en el momento de examinar si se conforma un régimen regional en salud subsiguiendo los desarrollos del CSS. Además, en la teoría neoliberal las instituciones son un instrumento valioso de los regímenes internacionales para lograr los propósitos de la cooperación (Jervis, 1999).

Todos estos patrones o indicios de institucionalización del tema de la salud en UNASUR fueron dinamizados por las organizaciones que se crearon, entiéndase por organizaciones el CSS y en un sentido más formal el Instituto de Salud Suramericano, este último se convirtió en la herramienta fundamental de acción del CSS. De tal suerte que la creación de estas organizaciones también hizo parte de la institucionalización del tema de la salud. Por lo anterior es necesario definir qué se entiende por organizaciones internacionales.

Las Organizaciones Internacionales (OI) "han jugado un rol fundamental no en algunas, sino en muchas, instancias de colaboración interestatal" (Abbot y Snidal, 1998, p.29). Las Ol emergen como instrumento central de la institucionalización de normas, acuerdos y procedimientos que han sido creados por el régimen internacional. Las OI tienen atributos funcionales (centralización e independencia), importantes para los estados, que motivan a estos últimos a preferirlas sobre otras formas de institucionalización y decidan actuar a través de ellas.

La centralización se entiende como la capacidad que tienen las organizaciones internacionales de dar soporte y centralizar actividades colectivas. Esto se logra por dos motivos: su concreta y estable estructura organizacional y su aparato administrativo. Cuando se cuenta con esta capacidad "incrementa la eficiencia de dichas actividades 
Revista Aportes para la Integración Latinoamericana Año XXIV, N³8/Junio 2018, ISSN 2468-9912. DNDA: 5355295 en línea

Milena Olivares Homez

El consejo de Salud Suramericano y la creación de un régimen regional de salud

Págs. 16-44

colectivas y a la vez va reforzando la habilidad organizativa, que influye directamente en acuerdos, entorno y en los intereses de los estados" (Abbott y Snidal, 1998, pp.4-5). En tanto la independencia se entiende como "la habilidad de actuar con un grado de autonomía dentro de esferas definidas, esto aumenta la capacidad para operar neutralmente en el manejo de las disputas y conflictos dentro de los estados" (Abbott y Snidal, 1998, p.5). Para el caso de estudio se evaluará si las organizaciones que se crearon al interior de UNASUR cumplen con estas características.

\section{Creación e institutcionalización del Consejo de Salud SuRamericano y sus ÓRGANOS SUBSIDIARIOS (GT, RES E ISAGS)}

\section{1. Creación e institucionalización del Consejo de Salud Suramericano}

Los países entendieron que la "la integración y la unión suramericanas son necesarias para avanzar en el desarrollo sostenible y el bienestar de nuestros pueblos, así como para contribuir a resolver los problemas que aún afectan a la región" (UNASUR, 2011). Esta propuesta de integración se hizo sobre la base del irrestricto respeto a la soberanía, integridad e inviolabilidad territorial de los Estados; autodeterminación de los pueblos; solidaridad; cooperación; paz; democracia; participación ciudadana y pluralismo; derechos humanos universales, indivisibles e interdependientes; reducción de las asimetrías y armonía con la naturaleza, principios que tuvieron un alcance mayor a los de cualquier otro proyecto integracionista que se haya dado en la región.

En 2008 se aprobó el Tratado Constitutivo de la UNASUR, que entró en vigencia en 2011 (UNASUR, 2011). En ese mismo año se aprobó el Estatuto de Funcionamiento y los Lineamientos Generales del Plan de Acción del CSS. A la creación del CSS concurrieron todos los países de la región en parte porque esta asistía a la pandemia de gripe AH1N1 y a las epidemias de Dengue y Cólera, que evidenciaron la necesidad de trabajar en conjunto para hacerles frente $y$, por otro lado, los países se habían comprometido a impulsar las decisiones que se tomaran en UNASUR.

Brasil y Ecuador le apostaron decididamente al tema de la salud por intereses propios. Brasil tiene la industria farmacéutica más potente de la región y podría vender los medicamentos que producía a un costo menor, cosa que beneficiaba a todos los países 
de la región, incluido Ecuador. Este último también tenía interés en la creación del CSS porque buscaba proteger la biodiversidad que es un recurso estratégico de desarrollo y que estaría mejor salvaguardado al interior de un bloque regional.

En abril de 2009, bajo la Presidencia Pro Tempore de Chile, se logra el Acuerdo Constitutivo № 01/09-21/04/2009 que le dará vida al CSS. Aquí se "establecen las atribuciones del Comité Coordinador, la Secretaria Técnica y la conformación de los Grupos Técnicos (GT), y se establecen las cinco áreas de trabajo del consejo, a saber: la Red Suramericana de Vigilancia y Respuesta en Salud; Desarrollo de Sistemas de Salud Universales; Acceso Universal a Medicamentos; Promoción de la Salud y Acción sobre los determinantes de la Salud; y, Desarrollo y Gestión de Recursos Humanos en Salud" (UNASUR, 2008). Estas áreas reflejaban el trabajo iniciado por la CAN, a través del ORAS-CONHU. Es importante señalar que las RES y el ISAGS no estaban contempladas en el Estatuto del CSS, pero aparecen inmediatamente después de la aprobación de este y se adicionan a la estructura del Consejo como parte fundamental de su operatividad, especialmente el ISAGS. De igual modo es necesario señalar que todos los desarrollos de estos órganos subsidiarios (RES, GT, ISAGS), deben contar con la aprobación del CSS y del Consejo de Jefas y Jefes de Estado como última instancia.

El CSS comenzó su accionar a finales de 2009 y a plenitud en 2010, con la formalización de su Plan Quinquenal 2010-2015 y, en este mismo año, se inició el debate del proyecto de creación del ISAGS. El plan quinquenal recoge las cinco áreas de trabajo y les provee de objetivos que constituyen las líneas de acción del CSS, a saber: 1) Establecer una red de Vigilancia Epidemiológica y Control de Enfermedades Transmisibles, No transmisibles y Eventos de Salud Pública 2) Formar Sistemas de Salud Universales en los países de Sudamérica reconociendo el derecho a la salud y el abordaje inclusivo 3) Desarrollar estrategias y planes de trabajo a fin de mejorar el acceso a medicamentos 4) Fortalecer la promoción de la salud y acción sobre los determinantes sociales con el objeto de reducir las inequidades en cada uno de los países y 5) Fortalecer la conducción, formulación, implementación y evaluación de políticas para el desarrollo y gestión de los recursos humanos de salud, en general y, en especial, en las áreas técnicas de la Agenda de Salud de UNASUR. EI Estatuto del CSS definió claramente los organismos de dirección y coordinación, en el siguiente gráfico se aprecia la estructura del CSS. 
Figura 1. Estructura del Consejo de Salud Suramericano

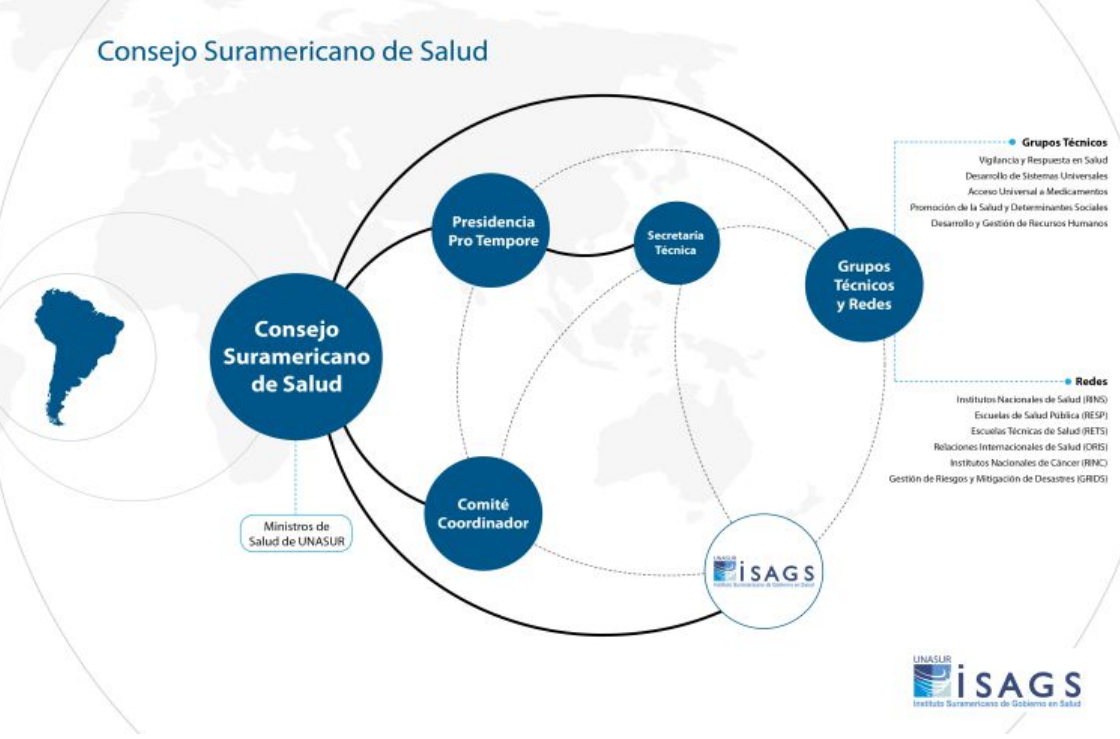

Fuente: ISAGS 2017

En el Estatuto del CSS (21 de abril de 2009) se le atribuyen las responsabilidades a los GT, siendo los "encargados de analizar, elaborar, preparar y desarrollar propuestas, planes y proyectos que contribuyan a ese propósito, de acuerdo con los lineamientos establecidos por el Plan Quinquenal (2010-2015). Cada grupo tiene un coordinador y un alterno con un mandato de dos años en total" (ISAGS, 2017a). Las coordinaciones iniciales de los GT, en el año 2009, fueron las siguientes:

Tabla 1. Coordinadores Grupos Técnicos del Consejo de Salud Suramericano Nombre del Grupo Técnico Coord. Principal Coord. Alterno 
Revista Aportes para la Integración Latinoamericana Año XXIV, N 38/Junio 2018, ISSN 2468-9912. DNDA: 5355295 en línea Milena Olivares Homez El consejo de Salud Suramericano y la creación de un régimen regional de salud Págs. 16-44

\begin{tabular}{|l|l|l|}
\hline Vigilancia y respuesta en salud & Paraguay & Uruguay \\
\hline Desarrollo de sistemas de salud universales & Chile & Bolivia \\
\hline Acceso universal a medicamentos & Argentina & Surinam \\
\hline $\begin{array}{l}\text { Promoción de la salud y determinantes } \\
\text { sociales }\end{array}$ & Venezuela & Colombia \\
\hline Desarrollo y gestión de recursos humanos & Brasil & Perú \\
\hline
\end{tabular}

Fuente: elaboración propia 2017

En relación con las Redes Estructurantes (RES), como se mencionó, no estuvieron en el Estatuto del CSS, se incorporaron tiempo después. Varios países de la región ya tenían redes en funcionamiento y lo que hace la UNASUR es incorporarlas al trabajo y a la estructura del CSS, en parte porque su experiencia era valiosa a la hora de articular temas específicos del área de la salud entre países. Peter Skerrett ${ }^{4}$ (en comunicación personal, 11 de abril, 2017), las definió como "un conjunto de experiencias focalizadas que agrupan las buenas prácticas de los estados, sintetizando, estas redes son la experiencia de los países compartiendo trabajo". Se decidió entonces conformar seis RES que estarían coordinadas a nivel individual por una Institución elegida por el Grupo Técnico de Recursos Humanos, cuyo mandato era de cuatro años. Las redes fueron "compuestas de instituciones designadas por los Ministerios de Salud de los 12 países miembros de UNASUR. Estas instituciones deben ser capaces de operar de manera eficaz, eficiente y sustentable los sistemas y servicios de salud a través de actividades Investigación \& Desarrollo y formación de personal" (ISAGS, 2017b).

Tabla 2. Redes Estructurantes

Red de los Institutos Nacionales de Salud (RINS)

Red de Escuelas Técnicas de Salud de UNASUR (RETS)

Red de Institutos e Instituciones Nacionales de Cáncer (RINC)

\footnotetext{
${ }^{4}$ Peter Skerrett, Director de Cooperación y Relaciones Internacionales del Ministerio de Salud Pública de Ecuador.
} 


\begin{tabular}{|l|}
\hline Red de Escuelas de Salud Pública de UNASUR (RESP) \\
\hline Red de Gestión del Riesgo de Desastres (RGRD) \\
\hline $\begin{array}{l}\text { Red de Oficinas de Relaciones Internacionales y de Cooperación Internacional en } \\
\text { Salud (REDSSUR-ORIS) }\end{array}$
\end{tabular}

\section{2. Institucionalización de los Grupos Técnicos y Redes Estructurantes}

Los acuerdos, informes y publicaciones que están en la web de la UNASUR y del ISAGS, dieron cuenta del avance en el trabajo de cada uno de los GT, que no fue homogéneo, en parte porque cada grupo estaba coordinado por un país diferente. En consecuencia, su ejecución estaba mediada por: 1) Las razones internas que predominaban en el sector de la salud y 2) Por la importancia que cada país le adjudicaba al trabajo de los equipos de salud, en el marco de las tareas orientadas por el CSS. Así, hubo una correspondencia evidente entre la capacidad de ejecución del trabajo de los GT y el estado que los dirigió. Tal como se observó en el caso de uno de los grupos que más se destacó: el grupo de Acceso Universal a Medicamentos, liderado por Argentina, país que en opinión de Breilh ${ }^{5}$ "posee una gran capacidad industrial y que ha permitido ahondar en investigaciones sobre la cuestión del acceso a los medicamentos" (comunicación personal, 4 de abril, 2017).

Este grupo creó el Banco de Precios de Medicamentos (BPM), con el apoyo del ISAGS. EI BPM es un instrumento que le proporciona información a los países sobre el valor de algunas medicinas en relación con las cantidades y con la compañía que las suministra, lo cual permite contrastar los precios y proveer de insumos a cada uno de los países para que mejore su capacidad negociadora con respecto a la multinacional, sin embargo, el uso del instrumento era discrecionalidad de los países. El BPM es una herramienta poderosa de negociación en bloque con las farmacéuticas, pero esto no se logró -al menos durante el periodo de estudio- porque no hubo consenso en los países para hacerlo. Sobre el BPM Carina Vance ${ }^{6}$ señaló que:

En estos momentos son alrededor de 140 medicamentos los que están en ese sistema y que básicamente ayudan a los países a tener información valiosa para los procesos de

\footnotetext{
5 Jaime Breilh, Médico y Rector de la Universidad Andina Simón Bolívar.

${ }^{6}$ Carina Vance, Directora Ejecutiva del ISAGS.
} 

Año XXIV, N 38/Junio 2018, ISSN 2468-9912. DNDA: 5355295 en línea

Milena Olivares Homez

El consejo de Salud Suramericano y la creación de un régimen regional de salud

Págs. 16-44

negociación de los precios en sus propios países; porque hay diferencias muy grandes entre país y país en relación con los precios a los que adquieren el mismo medicamento. Los países hicieron un ejercicio en noviembre del año pasado en el marco de la semana de la salud, donde de los medicamentos que habían incorporado al sistema hicieron un análisis de uno sobre la base del país que compró más barato ese medicamento y si toda la región adquiriera el medicamento al precio más bajo, la región podría ahorrar por año alrededor de 1.000.000 millones de dólares. (Comunicación personal, 26 de abril de 2017)

El grupo de Recursos Humanos también tuvo avances significativos en su trabajo y estaba coordinado por Brasil, sin duda ayudó su conocida trayectoria en los temas de capacitación e investigación en salud, que estaba en capacidad de ofrecer formación de alta calidad para los países de la región en materia de salud. Al respecto Breilh ${ }^{7}$ sostuvo que "Brasil tiene una amplia trayectoria en el tema de medicina social y fueron brasileños los que impulsaron el proceso de creación del ISAGS" (comunicación personal, 4 de abril, 2017). Este grupo ayudó en "la sistematización y difusión de las mejores prácticas y actividades de capacitación para el apoyo a funcionarios de Ministerios de Salud, Escuelas de Salud Pública y negociadores internacionales" (Riggirozzi, 2015, p.43).

En tanto los miembros de los grupos de trabajo de Vigilancia y Respuesta en Salud y Desarrollo de Sistemas de Salud Universales contribuyeron en la elaboración de las dos publicaciones que lanzó el ISAGS con relación a la vigilancia en salud en Suramérica y los sistemas de salud en la región.

Para estudiar el desempeño de las Redes Estructurantes se hizo necesario revisar sus publicaciones y además rastrear su nivel de actividad que estaba dado por las reuniones, coloquios, foros y conferencias que organizaron en torno a su tema. Las RES también fueron coordinadas de manera individual por diferentes países y de igual manera estuvieron a merced de las prioridades estatales en materia de salud. Sin embargo, durante el estudio se evidenció que el funcionamiento de las redes fue más intermitente que el de los grupos.

Del análisis de la información recopilada se sostiene que las redes más activas fueron: en primer lugar, la Red de Escuelas de Salud Pública, que está dirigida por la Escuela Nacional de Salud Pública de Brasil, que está vinculada a la FioCruz. En segundo lugar, la Red de Gestión del Riesgo de Desastres, la cual alterna su coordinación entre Chile y

\footnotetext{
${ }^{7}$ Jaime Breilh, Médico y Rector de la Universidad Andina Simón Bolívar.
} 
Perú y por último, está la Red de Institutos e Instituciones Nacionales de Cáncer (RINC), cuya secretaría técnica se estableció en el Instituto Nacional de Cáncer de Brasil.

La ubicación del ISAGS (Rio de Janeiro) permitió que las redes coordinadas por Brasil tuvieran un trabajo más dinámico. Como ejemplo de lo anterior se encontró que: "el Instituto y la Red -de Escuelas de Salud Pública- vienen trabajando de manera cercana desde el 2013 cuando asumieron juntos una serie de compromisos en el $3^{\circ}$ Foro Global sobre Recursos Humanos para la Salud, por ejemplo, la realización del Mapeo de la Oferta de Formación en Salud Pública en los países del bloque" (ISAGS, 2016, p.35).

Por su parte, la Red de Escuelas de Salud Pública organizó el III Coloquio Brasil-Cuba de Formación en Salud Pública, mientras que la Red de Institutos Nacionales de Cáncer, entregó un programa de prevención del cáncer de cuello uterino que incluye la vacuna contra el virus del Papiloma Humano y que Bolivia ya empezó a implementar ${ }^{8}$. La Red de RGRD generó el Plan de acción red de gestión del riesgo de desastres en salud del consejo de salud suramericano- UNASUR salud 2013 - 2021 y en el 2016 se inició la hoja de ruta para construir el Plan Suramericano de Riesgos de Desastres en Salud 20172021. En 2016 esta última red, junto con Ecuador, organizó la "Primera Reunión Regional de Alto Nivel sobre Gestión Inclusiva del Riesgo de Desastres".

En razón a lo expuesto anteriormente se infiere que las redes trabajaron principalmente en la organización de reuniones de coordinación y seminarios de discusión sobre los temas que cada una maneja. No obstante, y gracias al apoyo que brindó el ISAGS - y en algunos casos también en coordinación con los grupos técnicos-, alcanzaron productos específicos para la región, que son una fuente valiosa de información y de diagnóstico del área de la salud. Como ejemplo de lo anterior se tiene: el Plan Suramericano de Riesgos de Desastres en Salud y el programa de prevención de cáncer de cuello uterino, este último se viabilizó gracias al financiamiento del FIC (Fondo de Iniciativas Comunes) de la UNASUR.

\footnotetext{
${ }^{8}$ Veronica Zapana (5 de marzo de 2017). "Vacunarán a 81.000 niñas contra el papiloma humano en La Paz". Página Siete. Recuperado de http://www.paginasiete.bo/sociedad/2017/3/5/vacunaran-81000-ninas-contrapapiloma-humano-129483.html
} 

Año XXIV, N³8/Junio 2018, ISSN 2468-9912. DNDA: 5355295 en línea

Milena Olivares Homez

El consejo de Salud Suramericano y la creación de un régimen regional de salud

Págs. 16-44

\section{3. Creación e institucionalización del Instituto Suramericano de Gobierno en}

\section{Salud (ISAGS)}

En 2009, en la reunión del CSS realizada en Cuenca -Ecuador-, se aprobó la resolución que creó el ISAGS (también denominado Instituto de aquí en adelante) y las resoluciones que constituyeron su Consejo Directivo y Consultivo. El Instituto comenzó a funcionar temporalmente en el Centro de Relaciones Internacionales en Salud (CRIS) de la Fundación Oswaldo Cruz, en Rio de Janeiro y en 2012 el Consejo de Jefas y Jefes de Estado y de Gobierno de UNASUR, aprobaron el Estatuto del ISAGS, "fruto del consenso político alcanzado por los países de la región que reconocieron en la salud un puente hacia la paz y el desarrollo de los pueblos" (UNASUR, 2011a).

En 2012, se aprobó el Plan Trienal 2012-2015 donde se señala que el objetivo del ISAGS es "Constituirse en un centro de altos estudios y debate de políticas para el desarrollo de líderes y de recursos humanos estratégicos en salud, apuntando a fomentar la gobernanza y conducción en salud de los países de Sudamérica y dando insumos para articular la actuación regional en salud global” (ISAGS, 2012, p. 6). Con relación a esto su directora ejecutiva sostuvo: "estamos trabajando como un centro de pensamiento estratégico, tenemos modalidades de realizar talleres seminarios, también trabajamos en los temas que priorizan los países y tenemos productos como libros y proyectos específicos" (Carina Vance, comunicación personal, 26 de abril, 2017). Sin duda es una importante labor la que desempeña el Instituto que nació con el ánimo de "incentivar el debate democrático pensando en los derechos de las poblaciones y que pretendía estar más cercano al tejido social y a las comunidades de base, buscando convertirse en un motor de talleres de análisis en los países" (Jaime Breilh, comunicación personal, 4 de abril 2017).

Con la firma del acuerdo de ratificación de la sede del Instituto en Rio de Janeiro (mayo de 2016) se concluyeron las etapas formales de la institucionalización del ISAGS, se estableció su funcionamiento y se consolidó como una entidad intergubernamental, a la que se le dio derechos y funciones diplomáticas internacionales (Carina Vance, comunicación personal, 26 de abril de 2017). La aprobación del Plan de Trabajo Trienal del Instituto (2012-2015), el presupuesto para 2013, la distribución de cuotas entre los países miembros para el funcionamiento del instituto y la adopción de una guía de 
Revista Aportes para la Integración Latinoamericana Año XXIV, N³8/Junio 2018, ISSN 2468-9912. DNDA: 5355295 en línea

Milena Olivares Homez

El consejo de Salud Suramericano y la creación de un régimen regional de salud

Págs. 16-44

contratación, se entendieron como parte del proceso de institucionalización del ISAG. Finalmente, el define al instituto como una organización internacional, explicitando su alcance y capacidades.

Desde el inicio de su labor el ISAGS participó en las reuniones de las RES, los GT e instituciones que tienen que ver con la salud a nivel regional, siendo un puente entre las mismas y ayudando a coordinar acciones. Se convirtió en un espacio de encuentro y de apoyo en el trabajo de los grupos y las redes. Como ejemplo de esta coordinación de trabajo está el Banco de Precios de Medicamentos creación del Grupo de Trabajo de Acceso Universal a Medicamentos (GAUMU) que tuvo apoyo del ISAGS y que es una herramienta que les permite a los países mejorar su poción negociadora con las multinacionales farmacéuticas.

Todos los talleres y conferencias que realizó el instituto hasta 2017, apuntaron a fortalecer los temas que están enmarcados en las cinco áreas de trabajo del CSS. Todas estas actividades casi siempre se realizaron con organismos intergubernamentales que nutrieron la discusión y en un momento determinado sirvieron de apoyo para las acciones y planes que emanaban del CSS. Las actividades del instituto contaron con la presencia de las siguientes organizaciones: el Organismo Andino de Salud - Convenio Hipólito Unanue (ORAS-CONHU) y el Sector de Desarrollo en Salud de la Comunidad del Caribe (Caricom), la Universidad de las Naciones Unidas (UNU/CRIS), el Consejo de Desarrollo Social de UNASUR, Centro Rio+, Organización Panamericana de la Salud (OPS/OMS), Asociación Brasileña de las Industrias de Química Fina, Biotecnología y sus Especialidades (ABIAFINA), Instituto de Excelencia en Salud y Servicios Sociales (INESSS) de Quebec, en Canadá (ISAGS, 2015).

La información generada y recopilada por el Instituto se encuentra consignada en tres publicaciones (Periodo 2012-2015), en las cuales participaron todos los ministerios de América Latina y que presentan un panorama exhaustivo de la salud en la región. El ISAGS ha cumplido con el objetivo de convertirse en un centro de altos estudios un thinktank de la salud a nivel regional, así lo afirma Riggirozzi:

En conjunto con los Grupos de Trabajo, el ISAGS -con sede en Río de Janeiro- actúa como think-tank para la coordinación de la investigación relacionada con estos objetivos, formación de cuadros técnicos, y apoyo en vísperas de negociaciones internacionales, en el marco de la Organización Mundial de la Salud (OMS) (Riggirozzi, 2015, p.42). 
Revista Aportes para la Integración Latinoamericana Año XXIV, N³8/Junio 2018, ISSN 2468-9912. DNDA: 5355295 en línea

Milena Olivares Homez

El consejo de Salud Suramericano y la creación de un régimen regional de salud

Págs. 16-44

Otra de las labores más importantes del Instituto es su ayuda para lograr posiciones conjuntas de los países de la región en escenarios internacionales. Así se evidenció en el Cairo 2014, COP5 y, se acordaron intervenciones comunes para llevar al seno de la Asamblea № 69 de la OMS. De esta manera, se puede decir que el manejo del ISAGS ha significado un aporte valioso para proyectar a la región como un actor mundial, además, ha incidido en el tema de la salud de los ciudadanos a través de la participación constante y propositiva ${ }^{9}$ en los encuentros multilaterales. Así, Riggirozzi lo ratifica en sus investigaciones cuando indica que la "UNASUR ha estado actuando con una sola voz en la Asamblea Mundial de la Salud, presentando propuestas de reforma para mejorar la representación, gestión y forma (global) en que se definen los problemas de salud y las prioridades en la agenda internacional" (Riggirozzi, 2015, p. 44).

En suma, este apartado sostiene que durante el período estudiado el Instituto alcanzó el objetivo señalado en el Plan Trienal, el más importante de ellos: consolidarse como un centro de pensamiento de donde los gobiernos puedan nutrirse para sus propias políticas. El trabajo de los GT y las RES fue importante en el logro de este objetivo y en general estos organismos contribuyeron a fortalecer el papel del CSS como institución de consenso y determinación de políticas de salud a nivel regional.

\section{DESARROLLOS EN SALUd A LA LUZ DE LOS RegímENES INTERNACIONALES}

En este apartado se comparan vis a vis los resultados del proceso de institucionalización del CSS (incluyendo organismos subsidiarios) y las características de los regímenes regionales a la luz de la teoría neoliberal. Para indagar sobre la necesidad de creación del CSS es necesario mencionar a Stein cuando sostiene que "independientemente del país que se trate, todos tienen que afrontar dilemas de aversiones e intereses comunes y que los regímenes resuelven los primeros a través de la coordinación y los segundos por medio de la cooperación (Stein, 1982, pp.311-314).

En primera instancia, cabe señalar que el origen del CSS se ajustó a las motivaciones que dan origen a los regímenes que comparten intereses comunes, por dos motivos. El primero porque fue un deseo de todos los países comenzar a trabajar en el área de la

\footnotetext{
${ }^{9}$ UNASUR presentó una posición unificada, pidiendo a los países en desarrollo que tomaran conciencia del riesgo de impedir la expansión de la política de genéricos, tan importante para superar las barreras de acceso a los medicamentos (Agencia Fiocruz, 2012).
} 
salud y crear estrategias comunes para aliviar y mejorar la difícil situación en cada uno de los países de la región. El segundo fue la contingencia política dado tanto por el ascenso de los gobiernos de izquierda en la región como por las epidemias de cólera y gripe AN1H1 que obligó a los países a ver el tema de la salud como un tema de atención y respuesta común.

En segunda instancia y con relación a los incentivos, se encontró que dada la interdependencia entre los países de la región se aceptó la necesidad de buscar formas de acción conjunta para disminuir la pobreza y así mejorar el margen de maniobra de la región en el ámbito internacional. También se vieron como incentivos: la optimización de la acción regional frente a los convenios firmados con las multinacionales farmacéuticas y el impulso para la fabricación regional de medicamentos. Sin embargo, fueron los intereses de los países los que permitieron converger en este campo concreto.

Con relación a lo anterior es necesario señalar que los logros y adelantos que tuvo hasta ese momento la UNASUR en el ámbito de la salud fueron el resultado de un proceso de coordinación de expectativas y de un esfuerzo por recopilar datos reales y precisos sobre la situación de la salud en general, en toda la región. Esta captación de información es de vital importancia dado que, con ella y en concordancia con los fines Macro -de UNASURy micro -del CSS-, los mandatarios optarán por definir los temas de política pública. Temas que tendrán repercusión en la población de toda Suramérica. Una particularidad de los regímenes es que poseen la facultad de cooperar y coordinar en los campos de interés y aversión comunes, así los estados creerán en el régimen y evitarán realizar acciones individuales en esta área.

Como tercera instancia conviene evaluar al CSS como una organización internacional, y por tanto debe cumplir con los dos atributos funcionales que poseen las mismas (centralización e independencia). Atributos que hacen que en situaciones específicas los estados las prefieran para alternar formas de institucionalización y actuar a través de ellas, por ejemplo, las situaciones contingentes de las que se habló anteriormente. Los dos atributos son concurrentes en la institucionalidad que el tema de la salud tiene al interior de la UNASUR.

Con relación a la centralización, el CSS se constituyó como un órgano de información, unanimidad y cooperación en asuntos de la salud, con el fin de posicionar a "Sudamérica 
Revista Aportes para la Integración Latinoamericana Año XXIV, N 38/Junio 2018, ISSN 2468-9912. DNDA: 5355295 en línea

Milena Olivares Homez

El consejo de Salud Suramericano y la creación de un régimen regional de salud

Págs. 16-44

como un espacio de integración en salud que contribuya a la Salud para todos y al desarrollo, incorporando los esfuerzos y logros subregionales de MERCOSUR, Organismo Andino de Salud Convenio Hipólito Unanue y la Organización del Tratado de Cooperación Amazónica" (UNASUR, 2008). El CSS buscó coordinar acciones y generar estándares estatutarios viables para que los estados pudieran ejecutarlos simultáneamente, concentrando las obligaciones que se relacionaban con el monitoreo de la salud y posicionamiento del tema a nivel regional. De igual manera, formó una institucionalidad para gestionar sus propósitos, razón por la cual creó el ISAGS.

Por su parte, la independencia del CSS frente a intervenciones de gobiernos, estaba dada por dos circunstancias. Por un lado, todas sus actuaciones tenían que ser acordes con sus objetivos centrales y por otro, dichos objetivos hacían parte de los propósitos macro de cooperación de la UNASUR; de tal suerte que todo lo que se planificaba y ejecutaba estaba orientado a la consecución de dichos propósitos. Cabe mencionar aquí una anécdota que da cuenta del grado de independencia, Carina Vance señaló que en noviembre de 2016 se llevó a cabo la semana de la salud en UNASUR y en marzo de 2017 asistieron todos los ministros de salud de la región, a la cita con los -otroracandidatos a la Dirección General de la OMS, pese a que algunos países de la región tenían tensiones diplomáticas. También señalaba que los países siguieron cooperando conjuntamente en los proyectos que tenían con el ISAGS. Lo anterior evidenció una característica de los regímenes, que es la capacidad de operar neutralmente en el manejo de las disputas, Abbott y Snidal (1998).

Por lo señalado hasta aquí, conviene pensar en la significativa labor de investigación y mapeo regional que ha elaborado el Consejo de Salud Suramericano y sus respectivos órganos subsidiarios, que podrían guiar a los países en la toma de decisiones políticas en torno a la salud y por otro, les va generando un grado de confianza en la institucionalidad del CSS. A pesar de que es conocida la diferencia ideológica al interior de los países de Suramérica, se puede decir que una gran parte de estos halla en el CSS una manera de alcanzar sus objetivos en el tema de salud. Puede ser desde la efectividad del ahorro por medio de los descuentos en los costos de las medicinas y la ejecución de campañas públicas preventivas de salud hasta el alcance de un sistema universal de salud. 
En cuarta instancia se señala el tema de la reducción de costos de transacción que supone el régimen hacia sus miembros. En la medida en que el ISAGS se está convirtiendo en un organismo capaz de brindar información exacta sobre la salud regional en todas sus aristas, por ejemplo, los mecanismos de negociación de medicamentos o las políticas públicas de salud de algún país frente a una enfermedad regional; igualmente va realizando una de las funciones de los regímenes internacionales, que tal como señala Keohane (1982, p.338), reside en "mejorar la cantidad y la calidad de la información disponible para los actores", impidiendo que los estados determinen y resuelvan situaciones a nivel individual, lo que elevaría los costos de transacción.

Según Keohane, los regímenes internacionales posibilitan la instauración de acuerdos mutuos que son fundamentales en la creación -y soporte- de marcos de negociación para coordinar las expectativas de los actores (Keohane, 1982, pp.332-339). Ese rasgo de los regímenes puede ser entendido como la posibilidad que otorga el ISAGS de concertar posiciones y perspectivas conjuntas de los países, con el fin de optimizar las condiciones para la región.

Adicional a lo anterior, el Instituto "también puede ser usado para las investigaciones de cada país, lo que permitirá reducir los costos (de transacción en este caso) evitando contratar otros organismos para investigar" (Mariano Nascone ${ }^{10}$, comunicación personal, 10 de marzo de 2017). Esta capacidad de reducir los costos de transacción sigue siendo una función de los regímenes internacionales "los regímenes pueden hacer acuerdos fácilmente si -entre otras cosas- reducen otros costos de transacción, tales como costos de organización o de hacer pagos secundarios" (Keohane, 1982, p.338).

En razón a lo anterior puede sostenerse que el principal papel del ISAGS en conjunto con las RES y los GT fue el de proveer marcos de información precisa y completa sobre el tema de la salud en la región. De igual forma, contribuyeron en la consolidación de la región en ámbitos políticos, concertando posturas de los ministerios a escala regional para poder ser expuestas en las asambleas de la OMS. Por tal razón se señaló en su momento que en la UNASUR el área de la salud emergió como un "sitio para construir consensos, foco para formar cuadros políticos y técnicos y coordinador industrial,

\footnotetext{
${ }^{10}$ Mariano Nascone, Director de Asuntos Sociales de la UNASUR.
} 

Año XXIV, N 38/Junio 2018, ISSN 2468-9912. DNDA: 5355295 en línea

Milena Olivares Homez

El consejo de Salud Suramericano y la creación de un régimen regional de salud

Págs. 16-44

rompiendo con la tradicional transferencia pasiva de conocimientos y tecnologías desde países desarrollados hacia la región" (Riggirozzi, 2015, p.45).

En este apartado se expuso en detalle la forma en que trabajó el CSS en conjunto con los GT, las RES y el ISAGS. Desde el punto de vista de los regímenes existieron los incentivos para su creación y se dieron algunas de sus funciones. Sin embargo, no se aprecia ningún método de coerción que obligue a los países a cumplir los acuerdos o llevar a cabo las tareas conjuntas que se proponen o adoptar las recomendaciones que emanan del CSS en materia de salud, dado el funcionamiento de la UNASUR. Todo lo que se discute en esta organización debe aprobarse por consenso o de lo contrario no se adopta. De este modo no se contempla ningún mecanismo que le permita a ninguno de sus consejos tomar correctivos o algún tipo de sanción frente a algún Estado que no cumpla con lo acordado, es decir se está a merced de la "buena fe" y disposición de los países para trabajar mancomunadamente y ejecutar lo pactado.

\section{IV.CONCLUSIONES}

La situación contingente de América Latina en el momento en que aparece la UNASUR propició la creación del CSS, para tramitar los intereses comunes (mejorar la salud regional y lograr mejor acceso a medicamentos para combatir enfermedades), así como las aversiones: epidemias que traspasan fronteras y necesitan contestación regional. Desde este punto de vista, se cumpliría con los incentivos que permiten crear regímenes internacionales.

La creación del CSS y su funcionamiento como una organización internacional que provee centralización e independencia les permitió a los países confiarle al consejo muchas de sus investigaciones sobre salud, confianza generada gracias al trabajo de los órganos subsidiarios. Fue muy importante el apoyo que prestaron los GT, las RES y el ISAGS al CSS, entre los tres lograron darle sincronía al trabajo de la salud y permitieron articular los esfuerzos que hicieron otras organizaciones regionales en la misma área, de tal modo que no se duplicaran esfuerzos y fuesen más efectivas las acciones que en esta área se llevaron a cabo.

La institucionalidad que tiene el CSS, incluyendo la de sus órganos subsidiarios ha ayudado a consolidar acciones específicas que mejoran la condición negociadora de los 

Año XXIV, N 38/Junio 2018, ISSN 2468-9912. DNDA: 5355295 en línea

Milena Olivares Homez

El consejo de Salud Suramericano y la creación de un régimen regional de salud

Págs. 16-44

estados, por ejemplo, el BPM. Ante estos avances, los países están dispuestos cada vez más a colaborar con el CSS, esto también expresa su voluntad política. No obstante, no todo lo que se propone desde el CSS es adoptado decididamente por los países de la región, lo que no significa que no se haya avanzado. Tal vez aún no existe una resolución del CSS sobre muchos temas de la salud, sobre los cuales los órganos subsidiarios han venido trabajando, pero lo cierto es que todos los países de la región han empleado la información que aquellos han elaborado y en diferentes maneras han producido una sinergia con el Instituto, las Redes y los Grupos, y continúan formando parte del convenio macro de conseguir mejores condiciones de salud para todos los habitantes de Suramérica.

Los órganos subsidiarios tuvieron una importancia operativa en el cumplimiento de los objetivos del CSS, sin embargo, y debido a la necesidad de aprobar todo por consenso, algunos de sus desarrollos como el BPM no alcanzaron toda la efectividad que hubiesen podido. Aquí es donde se denota la falta de un instrumento de coerción u obligatoriedad que presione a los países, entre otras cosas, a adoptar acciones concretas frente a situaciones determinadas. Esta posibilidad de coerción o sanción a los países que incumplan las normas es una característica muy importante de los regímenes que hace que los países prefieran actuar a través de ellos en la arena internacional.

Frente a esta carencia señalada, la opción inmediata puede ser confiar en que los procesos de cooperación en salud que han logrado los países gracias al CSS, sean un incentivo que los obligue a seguir en esos acuerdos, más que salirse y afrontar las amenazas de salud a nivel individual; lo que no garantizaría mejores resultados que los que ofrecen estar dentro de los acuerdos.

En consecuencia, es necesario señalar que el caso de estudio denota características de regímenes en lo concerniente a los incentivos para su creación e incluso en sus etapas iniciales, pero para su cimentación y mantenimiento no está el elemento clave de tener mecanismos que persuadan a los miembros, en este caso los países de la región, a comportarse con arreglo a los fines pactados. Todos los acuerdos y posiciones políticas pactadas en la UNASUR salud quedan a discrecionalidad de cada uno de los países, se depende en gran medida de su voluntad política y en ese caso no prospera la función 

Año XXIV, N 38/Junio 2018, ISSN 2468-9912. DNDA: 5355295 en línea

Milena Olivares Homez

El consejo de Salud Suramericano y la creación de un régimen regional de salud

Págs. 16-44

esencial de los regímenes que es brindar confianza a sus miembros, confianza sobre las actuaciones de los otros en determinadas circunstancias.

Finalmente, es necesario precisar que puede ser cuestión de tiempo y de más compromiso político, el que se desarrolle un régimen regional en salud a plenitud, dado que ya se cuenta con varias características importantes y fundamentales para constituirlo. El germen ya está instalado y de continuar afianzando la labor de estos organismos, bajo la dirección del CSS es muy probable que dentro de algún tiempo pueda hablarse de prácticas-articuladas en políticas y acuerdos regionales en el área de la salud.

\section{BIBLIOGRAFÍA}

Abbott, K., \& Snidal, D. (1998). Why States Act through Formal InternationalOrganizations. Journal Of Conflict Resolution, 42(1), 3-32. Doi: $10.1177 / 0022002798042001001$

Aggarwal, V. (Marzo de 1981). Regime Transformation: An Analysis of the International Textile/ Apparel System-1955-1978. En International Studies Association, Filadelfia, Estados Unidos.

Balassa, B. (1964). The theory of economic integration. London: Routledge.

Briceño Ruiz, J. (Ed.) (2014). Del Regionalismo abierto al Regionalismo Post hegemónico en América Latina. Política Internacional e Integración Regional Comparada en América Latina., Costa Rica: UNA/FLACSO.

Frederking, B., \& Diehl, P. (2015). The politics of global governance (5th ed.). London: Lynne Rienner.

Hass, E. (Ed.) (1971). The Study of Regional Integration: Reflections on the Joy and Anguish of Pretheorizing. En Leon N. Lindberg y Stuart A. Scheingold. Regional Integration:Theory and Research, (pp 3-44). Cambridge, MA: Harvard University Press.

Haas, E. (1982). Words can hurt you; or, who said what to whom about regimes. International Organization, 36(2), 207-243. doi:10.1017/S0020818300018932

Hasenclever, A.Mayer, P. y Rittberger, V. (1997). Theories of International Regimes. Cambridge, Inglaterra: Cambridge University Press. 
Revista Aportes para la Integración Latinoamericana Año XXIV, N 38/Junio 2018, ISSN 2468-9912. DNDA: 5355295 en línea Milena Olivares Homez El consejo de Salud Suramericano y la creación de un régimen regional de salud Págs. 16-44

Instituto Suramericano de Gobierno en Salud ISAGS. (2012). Plan de Trabajo Trienal 2012-2015. Quito, Ecuador: UNASUR Recuperado de http://isagsunasur.org/es/documento/plan-de-trabajo-trienal-del-isags-2012-2015

(2015). ISAGS realiza V Reunión Ordinaria del Consejo Consultivo. Rio de Janeiro, Brasil: ISAGS. Recuperado de http://isagsunasul.org/it materia.php?ev=3060\&lg=2

(2016). Informe Anual del ISAGS 2016. Rio de Janeiro, Brasil: ISAGS. Recuperado de http://isags-unasur.org/es/informe-anual-del-isags-2016/

(2017a). Grupos Técnicos. Rio de Janeiro, Brasil: ISAGS. Recuperado de http://isags-unasur.org/es/unasul/grupos/

(2017b). Redes Estructurantes. Rio de Janeiro, Brasil: ISAGS Recuperado de http://isags-unasur.org/es/unasul/redes/

Jervis, R. (1982). Security Regimes. International organization. Volumen 36 (2), 357-378.

(1999). Realism, Neoliberalism, and Cooperation: Understanding the Debate. International Security. Volumen 24 (1), 42-63.

Keohane, R. (1982). The demand for International Regimes. International Organization. Volumen 36 (2), 325-355.

Keohane, R y Nye, J (ed). (2012). Interdependence in World Politics. En Power And Interdependence (3-22).Stoughton, Estados Unidos: Pearson.

Krasner, S. (1982a). Structural Causes and Regime Consequences: Regimes as Intervening Variables. International organization. Volumen 36 (2),185-205.

. (1982b). Regimes and the limits of realism. Regimes as Autonomous Variables.International organization. Volumen 36(2), 407-510.

Malamud, A. y Gardini, G. (2012). Has Regionalism Peaked? The Latin American

Quagmire and Its Lessons. The International Spectator: Italian Journal of International Affairs. Volumen 47 (1), 116-133.

Puchala, D. y Hopkins, R. (1982). Lessons from inductive analysis. International Organization. Volumen 36(2), 245-275. 
Revista Aportes para la Integración Latinoamericana Año XXIV, N³8/Junio 2018, ISSN 2468-9912. DNDA: 5355295 en línea

Milena Olivares Homez

El consejo de Salud Suramericano y la creación de un régimen regional de salud

Págs. 16-44

Riggirozzi, P. (Agosto de 2012a). Regional Health Governance in South America:

Redefining Regionalism and Regional Responsibilities. Ponencia presentada en la UNU/CRIS-UASB Doctoral School. Quito, Ecuador.

(2015). UNASUR: una construcción de una diplomacia regional en materia de salud a través de políticas sociales. Estudios Internacionales. (181), 29-51.

Riggirozzi, P. y Tussie, D. (2012). The Rise of Post - Hegemonic Regionalism. Londres, Inglaterra: Springer.

Rochester, J. (1975). International Institutions and World Power: The Intemational System as a Prismatic Polity. Beverly Hills, Estados Unidos: Sage.

Sanahuja, J. (2008-2009). Del 'regionalismo abierto' al 'regionalismo post-liberal' Crisis y cambio en la integración regional de América Latina. Anuario de la Integración regional de América Latina y el Caribe. (7),11-54.

(2012). Regionalismo post liberal y multilateralismo en Sudamérica: El caso de UNASUR. En Serbin, A, Martínez, L y Ramanzini. H (editores), El regionalismo"postliberal" en América Latina y el Caribe: Nuevos actores, nuevos temas, nuevos desafíos. Anuario de la Integración Regional de América Latina y el Gran Caribe 2012 (19-71). Buenos Aires, Argentina: CRIES.

Stein, A. (1982). Coordination and Collaboration: Regimes in an Anarchic World. International Organization. Volumen 36(2),299-324.

Unión de Naciones Suramericanas UNASUR. (2008). Decisión para el establecimiento del Consejo de Salud Sudamericano de la Unasur. Salvador de Bahía, Brasil: UNASUR. Recuperado de http://www.unasursg.org/es/documentos-normativos-unasur

(2011).Tratado Constitutivo de la Unión de Naciones Suramericanas. Quito, Ecuador: UNASUR. Recuperado de http://www.unasursg.org/es/documentos-normativos$\underline{\text { unasur }}$

(2011a). Estatuto del Instituto Suramericano de Gobierno en Salud. Buenos Aires, Argentina: UNASUR. Recuperado de http://www.unasursg.org/es/documentosnormativos-unasur 

Año XXIV, N 38/Junio 2018, ISSN 2468-9912. DNDA: 5355295 en línea Milena Olivares Homez El consejo de Salud Suramericano y la creación de un régimen regional de salud Págs. 16-44

Young, O. (1982). Regime dynamics: the rise and fall of International Regimes. International Organization, Volumen 36 (2), 277-297.

Olivares Homes Milena: Politóloga de la Universidad Nacional de Colombia y actual maestrante de Relaciones Internacionales con mención en Negociación y Cooperación Internacional de FLACSO-Ecuador. Sus líneas de investigación son regionalismo, regímenes internacionales y oriente medio. Ha publicado artículos académicos y de opinión sobre los temas en mención. Correo electrónico: milenaolivareshomez@gmail.com. 